\title{
Research Article Effect of Trends on Detrended Fluctuation Analysis of Precipitation Series
}

\author{
Jianhai Yue, ${ }^{1}$ Xiaojun Zhao, ${ }^{2}$ and Pengjian Shang ${ }^{2}$ \\ ${ }^{1}$ School of Mechanical, Electronic and Control Engineering, Beijing Jiaotong University, \\ Beijing 100044, China \\ ${ }^{2}$ Department of Mathematics, School of Science, Beijing Jiaotong University, Beijing 100044, China \\ Correspondence should be addressed to Xiaojun Zhao, 05271060@bjtu.edu.cn
}

Received 21 January 2010; Revised 29 March 2010; Accepted 26 April 2010

Academic Editor: Ming Li

Copyright (C) 2010 Jianhai Yue et al. This is an open access article distributed under the Creative Commons Attribution License, which permits unrestricted use, distribution, and reproduction in any medium, provided the original work is properly cited.

\begin{abstract}
We use detrended fluctuation analysis (DFA) method to detect the long-range correlation and scaling properties of daily precipitation series of Beijing from 1973 to 2004 before and after adding diverse trends to the original series. The correlation and scaling properties of the original series are difficult to analyze due to existing crossovers. The effects of the coefficient and the power of the added trends on the scaling exponents and crossovers of the series are tested. A crossover is found to be independent of the added trends, which arises from the intrinsic periodic trend of the precipitation series. However, another crossover caused by the multifractal vanishes with the increasing power of added trends.
\end{abstract}

\section{Introduction}

Many physical and biological systems exhibit complex behavior characterized by long-range power-law correlations. Traditional approaches such as the power-spectrum and scaledHurst analysis are limited to quantify correlations in stationary signals. In recent years, detrended fluctuation analysis (DFA) has been established as an important tool for the detection of long-range correlations in time series with nonstationarities. DFA is a scaling analysis method providing a quantitative parameter, the scaling exponent $\alpha$, to represent the long-range autocorrelation properties of a signal. The advantages of DFA over many other methods are that it permits the detection of correlations in apparent nonstationary time series and also avoids the spurious detection of seemingly long-range correlations that are artifact of nonstationarity. DFA which is a nonparametric approach for data mining has been successfully applied to diverse fields of interest such as DNA, heart rate dynamics, neuron spiking, human gait, cloud structure, economical time series, and long-time weather records as well as [1-9]. Besides, many parameter models as well as relevant prediction also have been systematically explored such as in traffic flows with remarkable results [10-14]. 
A fact exists that precipitation has a dramatic effect on agriculture and plays a significant role in human's activities. The study of precipitation can be utilized for several purposes, including hydrological structure design, flood prevention, and so forth. Precipitation has been long analyzed by traditional statistics, and effective methods as well as prediction models have been developed in bulk to investigate its role [15-17]. There also exist many investigations of scaling behaviors and multifractal characterization of the precipitation records [18-20]. However, traditional time series analysis of precipitation always produces spurious results due to the highly nonstationary nature of precipitation signals. Matsoukas et al. [21] used detrended fluctuation analysis to quantify the correlation properties of precipitation time series but did not describe them in detail.

In the paper, we detect the long-range correlations of the daily precipitation series collected from 21 weather stations of Beijing through about 30 years and investigate their correlation properties together with the influence of added trends under the method of DFA. As external trends are the main components which affect the correlation properties of a time series, people are trying to eliminate them to gain proper insight into the records. However, in most cases it is difficult to distinguish the trends from the intrinsic fluctuations in data. We add diverse trends on the contrary to the original data and systematically analyze their effect on the correlation properties. The essence of adding the trends in the paper is a preprocessing as the trends will be the functions of original series.

The organization of this paper is as follows: in Section 2, we briefly introduce the DFA method. Section 3 is about the details of the precipitation data we used in this paper. In Section 4 we detect the correlation properties by calculating the scaling exponents and crossover times of the original series before and after adding correlated trends. We summarize in Section 5.

\section{Methodology}

Experimental series are often affected by nonstationarity and fractality [22]. To investigate the scaling behavior of fluctuations, external trends are expected to be well distinguished from the intrinsic fluctuations of the system. If trends exist in the data, Hurst rescaled-range analysis and other nondetrending methods might give spurious results [5-8]. Very often we never know the reasons for underlying trends in collected data and even worse the scales of the underlying trends. DFA is a well-established and robust method for determining the scaling behavior of noisy data in the presence of diverse trends [17-21].

For a record $\{X(i)\}, i=1,2, \ldots, N$, where $N$ denotes the length of record, the DFA procedure briefly involves the following four steps.

Step 1. We determine the profile $x(i)$,

$$
x(i)=\sum_{k=1}^{i} X_{k}-\langle X\rangle
$$

where $\langle X\rangle$ is the mean of the record.

Step 2. We cut the profile $x(i)$ into $N_{s}=[N / s]$ boxes of the same size $s$. In each box, we fit the integrated time series by using a polynomial function, $p_{v}(i)$, which is regarded as the local 
trend. For order- $n$ DFA, $n$ order polynomial function is applied of the fitting approximation. We subtract the local trend in each box and get the detrended fluctuation function $x_{s}(i)$ :

$$
x_{S}(i)=x(i)-p_{v}(i)
$$

Step 3. In each box of size $s$, we calculate the root mean square (rms) fluctuation $F(s)$ :

$$
F(s)=\sqrt{\frac{1}{N_{s}} \sum_{i=1}^{N_{s}} x_{s}^{2}(i)} .
$$

Step 4 . We repeat this procedure for different box sizes $s$ (different scales).

If a power-law relation exists between $F(s)$ and $s$,

$$
F(s) \sim s^{\alpha} .
$$

It indicates the presence of scaling property. The parameter $\alpha$, called the scaling exponent or fluctuation exponent, represents the correlation properties of the data. For correlation exponent $\gamma$, which is derived from the autocorrelation function, a similar approximation for $F(s)$ is

$$
F(s) \sim s^{1-\gamma / 2} \text {. }
$$

Comparing with (2.4), we find $\gamma=2-2 \alpha$ for $0<\gamma<1$. A brief certification of the relation of $\gamma$ and $\alpha$ is proposed in [23]. We can determine the correlation exponent $\gamma$ by measuring the fluctuation exponent $\alpha$. If $\alpha=0.5$, there is no correlation (white noise); if $\alpha<0.5$, the data is anticorrelated; if $\alpha>0.5$, the data is long-range correlated.

\section{Data Description}

The precipitation data here is collected from 21 weather stations of Beijing from January 1 1973 to December 31 2004, 11688 days, as illustrated in Figure 1. A vision processing based on coded structure light has been investigated to acquire 3D data which can be referred for further analysis if necessary $[24,25]$. There may not be any precipitation record which is different from such as temperature series. There may be a little precipitation that it is not necessary for the weather stations to record in detail but adopting a word "minim" instead of a specific quantity. For convenience, we regard the quantity of the days without precipitation as 0 and the "minim" as 0.5 . We treat the mean value of the 21 records every day of different stations as a new precipitation series for analysis.

\section{Data Analysis}

\subsection{DFA of the Original Series}

First, we detect the correlation behavior of the original series. To get more information, we use the DFA arranging from 1st to 5th order. The original series is a multifractal according 


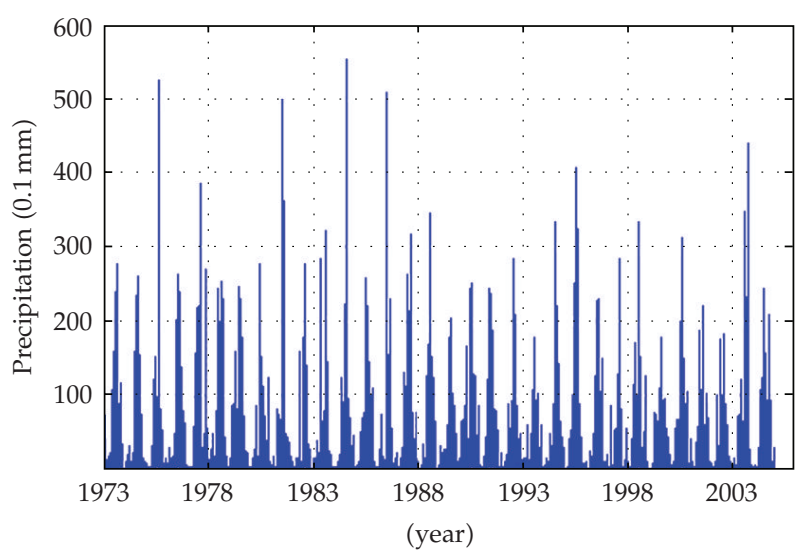

Figure 1: illustrates the precipitation data of every day in the record years. The unit of the precipitation is $0.1 \mathrm{~mm}$. From this figure, we can clearly see the seasonal trend of the data. We derive similar results from the DFA of the original data shown in next section.

to Figure 2(a) since the scaling exponents of each order- $n$ DFA change twice, that is, two crossovers. At small scales $s$, the deviations grow stronger with the increasing DFA order $n$. To decrease the impact of the deviations on the calculating of scaling exponents $\alpha^{(n)}$, we ignore some small $s$ while fitting the curve.

Crossover times $s_{1 x}^{(n)}$ and $s_{2 x}^{(n)}$ are determined by the intersection of linear fits done on both sides of the crossovers. We choose the point at the intersection in scales $\{s\}$ as the crossover times $s_{x}^{(n)}$, calculate the slope on both sides of $s_{x}^{(n)}$ to get $\alpha^{(n)}$, and exhibit $s_{x}^{(n)}, \alpha^{(n)}$ in Table 1.

$s_{1 x}^{(n)}$ and $s_{2 x}^{(n)}$ of each order- $n$ DFA divide the series into three different scaling segments. At the first segment where the time scales $s<s_{1 x}^{(n)}, 0.5<\alpha_{0}^{(n)}<1$ corresponds to a long-range correlation behavior which indicates that a relatively large magnitude is likely to be followed by a large magnitude event. For the two segments on the two sides of the second crossover $s_{2 x}^{(n)}$, scaling exponents $\alpha^{(n)}$ changes from $\alpha_{0}^{(n)}>1$ to $\alpha_{2}^{(n)}<0.5$ which means that at large time scales $s$, the series is anticorrelated. The scaling behavior of the original series at large scales $s$ is similar to the DFA of a sinusoidal series shown in Figure 2(b). Comparing Figure 2(a) with Figure 2(b), there exist some common properties at large scales; after a significant crossover, both scaling exponents turn rather small. It manifests that periodic trend dominates the scaling property at large scales after $s_{1 x}^{(n)}$ which is accordant to the investigations in [4]. It also can be referred that $s_{2 x}^{(n)}$ is highly possible to be dominated by the seasonal trend in the precipitation series.

\subsection{DFA of the Series with Correlated Trend $A[X(i)]^{p}$}

The complex properties of the original series result from the crossover times $s_{1 x}^{(n)}$ and $s_{2 x}^{(n)}$, so it is difficult to understand the scaling behavior and make a valid prediction. A crossover usually can arise from a change in the correlation properties of the series at different time scales, that is, multifractal, or can often arise from external trends in the data [26]. Diverse methods provide inspiration to produce discrete sequences and continuous functions [27, 28] 
Table 1: The crossover times $s_{x}^{(n)}$ and the scaling exponent $\alpha^{(n)}$ of the original data.

\begin{tabular}{lccccc}
\hline & DFA1 & DFA2 & DFA3 & DFA4 & DFA5 \\
\hline$\alpha_{0}^{(n)}$ & 0.6718 & 0.6263 & 0.6122 & 0.6318 & 0.6415 \\
$s_{1 x}^{(n)}$ & 84 & 98 & 146 & 234 & 274 \\
$\alpha_{1}^{(n)}$ & 1.1407 & 1.2772 & 1.3650 & 1.4857 & 1.4927 \\
$s_{2 x}^{(n)}$ & 406 & 556 & 704 & 892 & 965 \\
$\alpha_{2}^{(n)}$ & 0.1512 & 0.1419 & 0.1236 & 0.1263 & 0.1296 \\
\hline
\end{tabular}

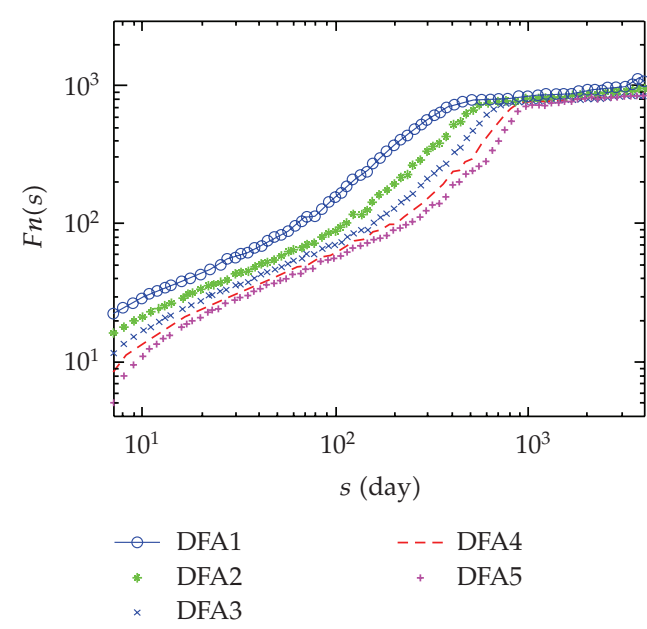

(a)

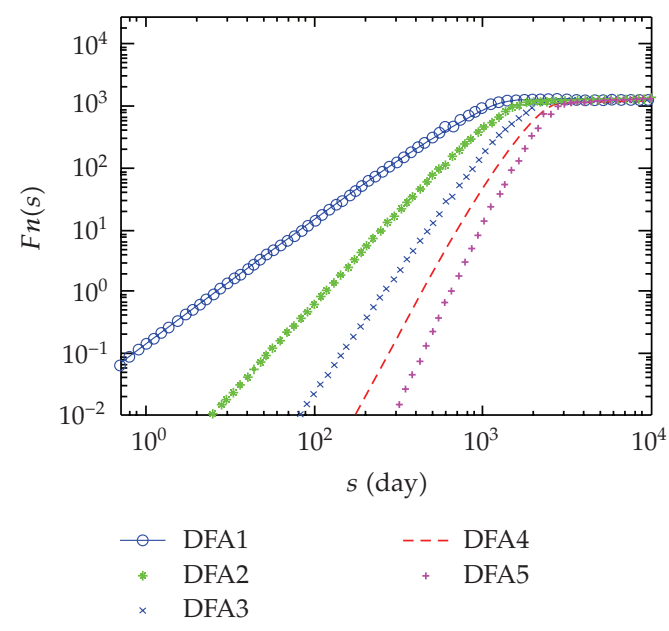

(b)

Figure 2: (a) DFA of the original series. For each order- $n$ DFA there exists two crossovers $s_{1 x}^{(n)}$ and $s_{2 x}^{(n)}$ (We use $s_{x}^{(n)}$ for them) which divide the curves into 3 different scaling segments whose scaling exponents are: $\alpha_{0}^{(n)}, \alpha_{1}^{(n)}$ and $\alpha_{2}^{(n)}$ respectively (We use $\alpha^{(n)}$ for them). (b) The DFA of a sinusoidal series given by the function $10 * \sin (20 \pi i / N)$, where $N$ is the length of the original series

for simulation. In most cases, people generate long-range correlated experimental data with modified Fourier filtering [29] or "ARFIMA" [30] method and superimpose diverse trends on them which are the function of time, like linear, sinusoidal, and power-law trend. The trends effects on the original series are tested, mainly including the crossover, complete with diverse detrending methods based on such as SVD [9], EMD [31-33], Fourier-DFA [34], wavelet analysis [35], and "superposition rule" [4]. In real data, the type of trend is analyzed and proper detrending method is employed correspondingly. It is an attractive and logical direction in solving the crossover caused by trends and deriving a constant scaling exponent. However, at times the type of the trend is difficult to identify, and we note that the information of the series is not fully uncovered just by the DFA method. Here we firstly propose a new method to preprocess the data by adding a trend which is a function of the original series. Then we test whether and how correlated trends added to the original series will affect the correlation properties. A common power-law function $A[X(i)]^{p}$ is used, where $A$ is a coefficient and $p$ presents the power. We apply DFA method to the new series $Y(i)=X(i)+A[X(i)]^{p}$. It is apparent that $Y$ share the same period of trend with $X$ but with 
Table 2: Scaling exponents $\alpha^{(n)}$ and crossover times $s_{x}^{(n)}$ of the series with correlated trends $A[X(i)]^{p}, A=1$ and $p$ are integers from 1 to 6 .

\begin{tabular}{|c|c|c|c|c|c|c|}
\hline & & DFA1 & DFA2 & DFA3 & DFA4 & DFA5 \\
\hline \multirow{5}{*}{$p=1$} & $\alpha_{0}^{(n)}$ & 0.6718 & 0.6263 & 0.6122 & 0.6318 & 0.6415 \\
\hline & $s_{1 x}^{(n)}$ & 84 & 98 & 146 & 234 & 274 \\
\hline & $\alpha_{1}^{(n)}$ & 1.1407 & 1.2772 & 1.3650 & 1.4857 & 1.4927 \\
\hline & $s_{2 x}^{(n)}$ & 406 & 556 & 704 & 892 & 965 \\
\hline & $\alpha_{2}^{(n)}$ & 0.1512 & 0.1419 & 0.1236 & 0.1263 & 0.1296 \\
\hline \multirow{5}{*}{$P=2$} & $\alpha_{0}^{(n)}$ & 0.6153 & 0.5927 & 0.5856 & 0.5966 & 0.6073 \\
\hline & $s_{1 x}^{(n)}$ & 106 & 116 & 146 & 234 & 274 \\
\hline & $\alpha_{1}^{(n)}$ & 0.8732 & 0.9452 & 0.9890 & 1.0643 & 1.0593 \\
\hline & $s_{2 x}^{(n)}$ & 406 & 556 & 704 & 892 & 965 \\
\hline & $\alpha_{2}^{(n)}$ & 0.2680 & 0.2256 & 0.1687 & 0.1583 & 0.1544 \\
\hline \multirow{3}{*}{$P=3$} & $\alpha_{1}^{(n)}$ & 0.6031 & 0.6021 & 0.6015 & 0.6026 & 0.6024 \\
\hline & $s_{2 x}^{(n)}$ & 406 & 556 & 704 & 892 & 965 \\
\hline & $\alpha_{2}^{(n)}$ & 0.3361 & 0.2772 & 0.2224 & 0.2151 & 0.2197 \\
\hline \multirow{3}{*}{$P=4$} & $\alpha_{1}^{(n)}$ & 0.5499 & 0.5570 & 0.5565 & 0.5600 & 0.5633 \\
\hline & $s_{2 x}^{(n)}$ & 406 & 556 & 704 & 892 & 965 \\
\hline & $\alpha_{2}^{(n)}$ & 0.3709 & 0.3198 & 0.3010 & 0.3008 & 0.3073 \\
\hline \multirow{3}{*}{$P=5$} & $\alpha_{1}^{(n)}$ & 0.5271 & 0.5367 & 0.5365 & 0.5407 & 0.5454 \\
\hline & $s_{2 x}^{(n)}$ & 406 & 556 & 704 & 892 & 965 \\
\hline & $\alpha_{2}^{(n)}$ & 0.3951 & 0.3538 & 0.3638 & 0.3672 & 0.3721 \\
\hline \multirow{3}{*}{$P=6$} & $\alpha_{1}^{(n)}$ & 0.5177 & 0.5276 & 0.5279 & 0.5322 & 0.5377 \\
\hline & $s_{2 x}^{(n)}$ & 406 & 556 & 704 & 892 & 965 \\
\hline & $\alpha_{2}^{(n)}$ & 0.4138 & 0.3760 & 0.4012 & 0.4060 & 0.4106 \\
\hline
\end{tabular}

different fluctuation magnitudes. As we will see later, $s_{2 x}^{(n)}$ dominated by periodic trend is independent of added trend $A[X(i)]^{p}$, but $s_{1 x}^{(n)}$ rises from the multifractal will disappear. We make $A$ and $p$ variables, respectively, to operate our study as follow.

\subsubsection{Effect of Power $p$ on DFA of $Y(i)$}

In this section, $p$ is a variable and $A$ is a constant 1 .

\section{(1) $p$ Is Positive Integer}

Considering the capability of order- $n$ DFA in removing trend of $(n-1)$ th order, we give $p$ integer values ranging from 1 to 6 to take a whole view of effects of DFA on the series $Y(i)$.

For $s<10^{3}$ every order- $n$ DFA in Figures 3(a) and 3(b), two crossovers exist while in (c) the crossovers only remain in DFA3, DFA4, and DFA5 together with their positions being much closer. For (d), (e), and (f), although the crossovers still exist, one cannot identify them 


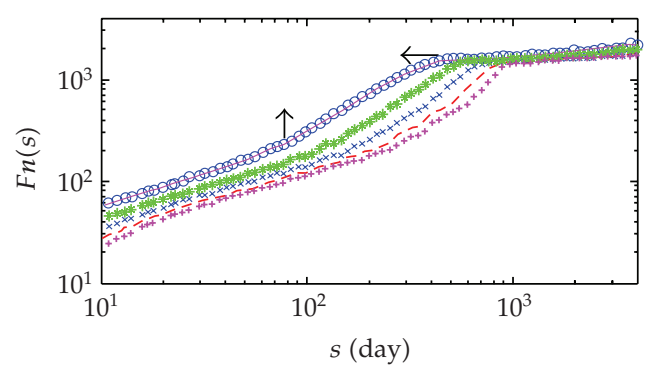

(a)

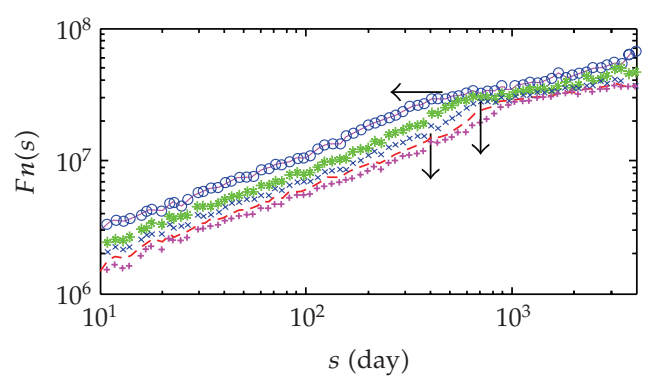

(c)

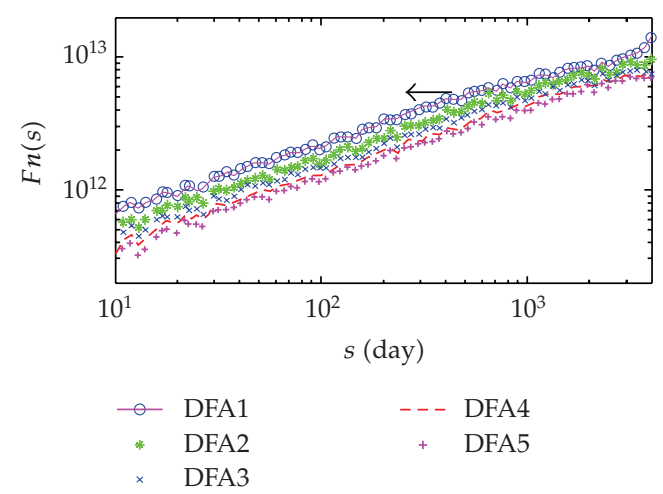

(e)

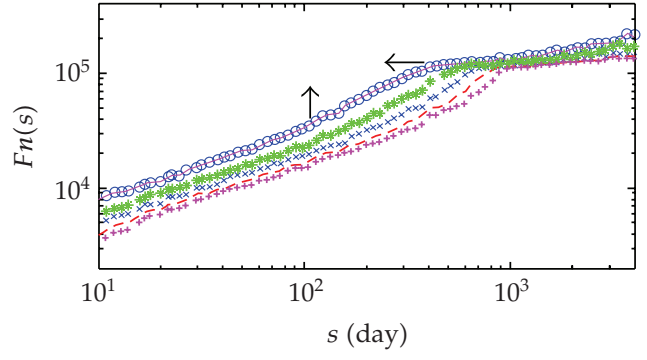

(b)

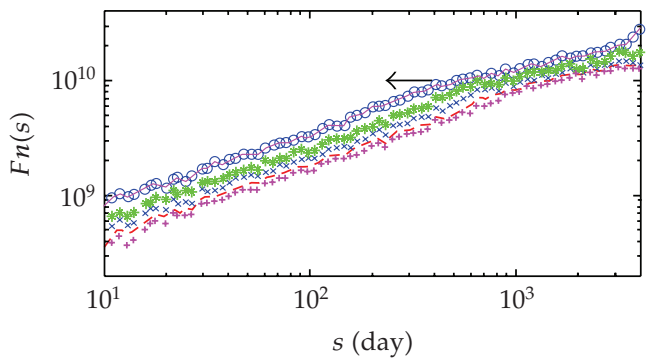

(d)

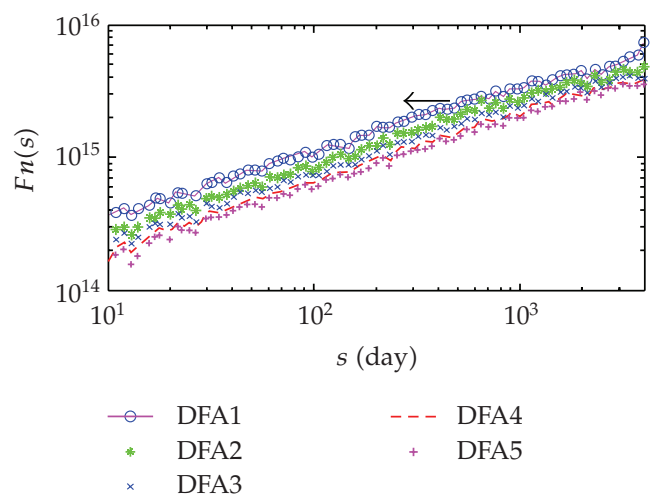

(f)

Figure 3: DFA of the series with correlated trend $A[X(i)]^{p}$, that is, $Y(i)=X(i)+A[X(i)]^{p}$, where $A=1$, (a) $p=1$, (b) $p=2$, (c) $p=3$, (d) $p=4$, (e) $p=5$, (f) $p=6$.

without checking carefully. For each order- $n$ DFA in (d), (e), and (f), only one crossover exists, which is still marked by only $s_{2 x}^{(n)}$ for convenience. We illustrate the representative crossovers by arrows. It seems that crossovers $s_{2 x}^{(n)}$ in all six subfigures share an identical position. We apply the method in Section 4.1, calculate these crossovers and scaling exponents, and specify the crossover time scales in Table 2. Since the scaling behavior of DFA3, DFA4, and DFA5 in Figure 3(c) on both sides of $s_{1 x}^{(n)}$ is just the same, we calculate one scaling exponent $\alpha_{1}^{(n)}$ for each of them before $s_{2 x}^{(n)}$ in Table 2 .

The positions of crossover times $s_{2 x}^{(n)}$ after adding diverse trends are identical to that of the original series, which demonstrate that $s_{2 x}^{(n)}$ are independent of the power $p$ of the correlated trend. With the increasing of $p$ from 1 to 6 , the values of all scaling exponents $\alpha_{0}^{(n)}$ 
Table 3: Comparison of $\alpha_{1}^{(n)}$ and only $\alpha_{2}^{(n)}$ from $p=1$ to $p=6$ after recalculating of $p=1$ and $p=2$.

\begin{tabular}{ccccccc}
\hline & & DFA1 & DFA2 & DFA3 & DFA4 & DFA5 \\
\hline \multirow{4}{*}{$\alpha_{1}^{(n)}$} & $P=1$ & 0.8775 & 0.8300 & 0.8238 & 0.8106 & 0.7885 \\
& $P=2$ & 0.7113 & 0.6924 & 0.6907 & 0.6862 & 0.6779 \\
& $P=3$ & 0.6031 & 0.6021 & 0.6015 & 0.6026 & 0.6024 \\
& $P=4$ & 0.5499 & 0.5570 & 0.5565 & 0.5600 & 0.5633 \\
& $P=5$ & 0.5271 & 0.5367 & 0.5365 & 0.5407 & 0.5454 \\
& $P=6$ & 0.5177 & 0.5276 & 0.5279 & 0.5322 & 0.5377 \\
\hline \multirow{4}{*}{$\alpha_{2}^{(n)}$} & $P=1$ & 0.1512 & 0.1419 & 0.1236 & 0.1263 & 0.1296 \\
& $P=2$ & 0.2680 & 0.2256 & 0.1687 & 0.1583 & 0.1544 \\
& $P=3$ & 0.3361 & 0.2772 & 0.2224 & 0.2151 & 0.2197 \\
& $P=4$ & 0.3709 & 0.3198 & 0.3010 & 0.3008 & 0.3073 \\
& $P=5$ & 0.3951 & 0.3538 & 0.3638 & 0.3672 & 0.3721 \\
& $P=6$ & 0.4138 & 0.3760 & 0.4012 & 0.4060 & 0.4106 \\
\hline
\end{tabular}

and $\alpha_{1}^{(n)}$ before $s_{2 x}^{(n)}$ decrease to 0.5 while all values of $\alpha_{2}^{(n)}$ after $s_{2 x}^{(n)}$ have the trend to increase to 0.5 . To get a clearer view of this phenomenon, for $p=1$ and $p=2$, we calculate a new scaling exponent $\alpha_{1}^{(n)}$ before $s_{2 x}^{(n)}$ of each order- $n$ DFA by linear fit. They are specified, respectively in Table 3.

The decreasing trend of $\alpha_{1}^{(n)}$ to 0.5 and the increasing trend of $\alpha_{2}^{(n)}$ to 0.5 are shown in Table 3. With the increasing of $p$ the approaching pace of $\alpha_{2}^{(n)}$ to 0.5 seems to be slower than that of $\alpha_{1}^{(n)}$.

$s_{1 x}^{(n)}$ vanishes with increasing $p$ of the trend $A[X(i)]^{p}$ but $s_{2 x}^{(n)}$ is independent of the added trend. It agrees well with the previous analysis that $s_{1 x}^{(n)}$ and $s_{2 x}^{(n)}$, respectively arise from the multifractal and periodic trend in precipitation series.

The scaling behavior of $Y(i)$ is similar to that of original signal when $p=1$, as $Y(i)=(A+1) X(i)$. When $p>1$, since the magnitude of $X(i)$ is mostly larger than 1 , the scaling properties of $A[X(i)]^{p}$ will play a vital role and the influence of $X(i)$ can be negligible which also can be inferred from the scale of the fluctuation $F_{n}(s)$. In fact, Figures 3(b)$3(\mathrm{f})$ demonstrate the fluctuations of $A[X(i)]^{p}$ as well. We note that the scaling properties of $A[X(i)]^{p}$ is also attractive, as it is the function of original series which can be treated a preprocessing.

\section{(2) $p$ Is Number with Decimal and $p>1$}

The crossovers $s_{2 x}^{(n)}$ in Figure 3 are independent of power $p$ but $s_{1 x}^{(n)}$ are not which is, respectively, affected by the multifractal and periodic trend of original precipitation series. Figures $3(\mathrm{~b}), 3(\mathrm{c})$, and $3(\mathrm{~d})$ show a "vanishing" process of $s_{1 x}^{(n)}$, where $s_{1 x}^{(n)}$ remains by adding an order- $p$ trend while disappears by an order- $p+1$ trend. However, we also expect to track the whole process of vanishing with a decimal of $p$. We test it in Figure 4 . First we find some common properties in three pair figures that these significant processes all take place between $m+0.8$ to $m+0.9, m=1,2,3$, which are very close to $m+1$. As we use DFA to 5 th order, the "vanishing" process of $s_{1 x}^{(n)}$ just completes when the power of the trend $A[X(i)]^{p}, p$, is 3.9, close to $5-1=4$. Thus we can make a hypothesis that if our DFA is order $n+1$, there will be some significant process around each $p=i+0.9, i=1,2, \ldots, n-1$, close to $i+1$ and we 


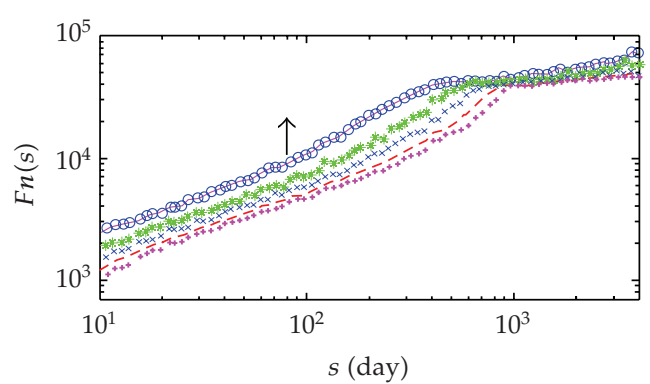

(a)

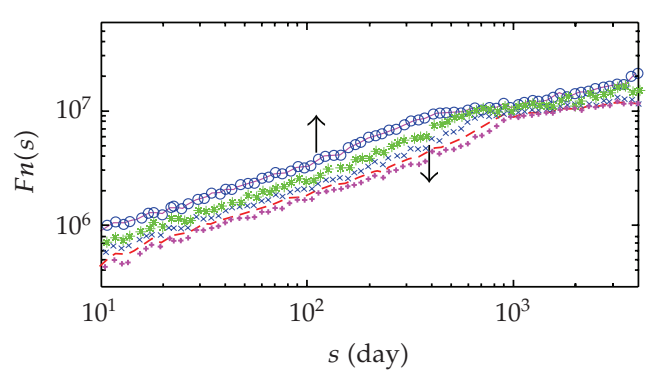

(c)

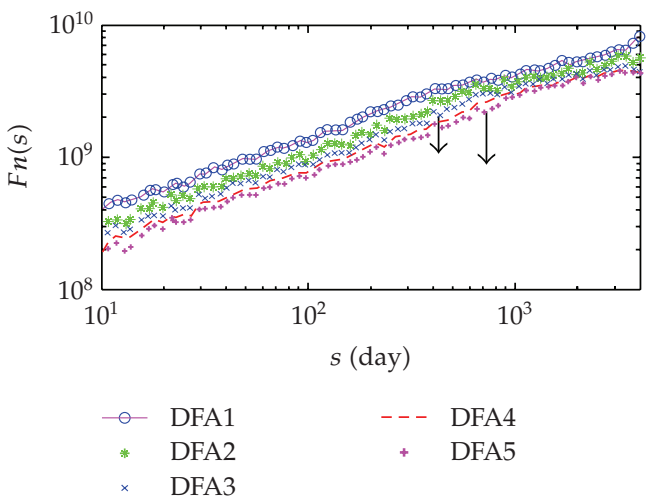

(e)

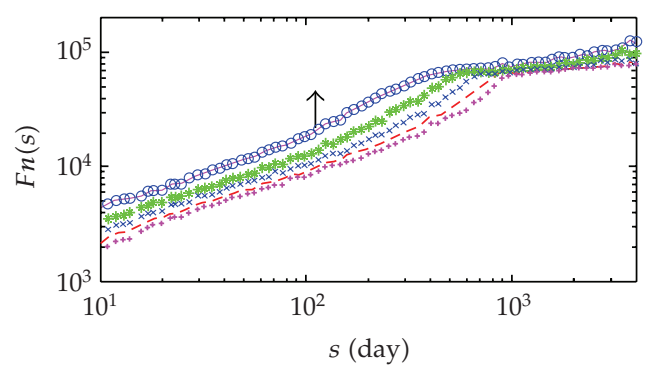

(b)

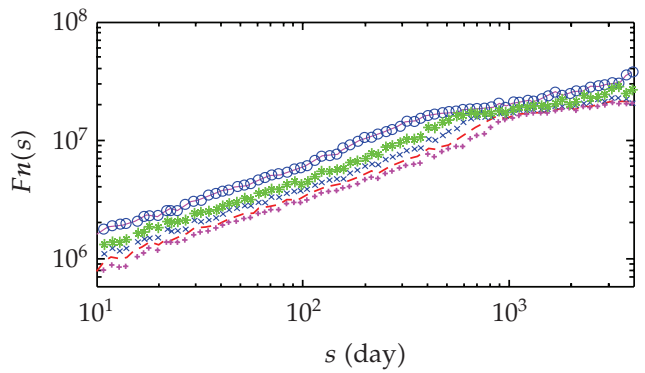

(d)

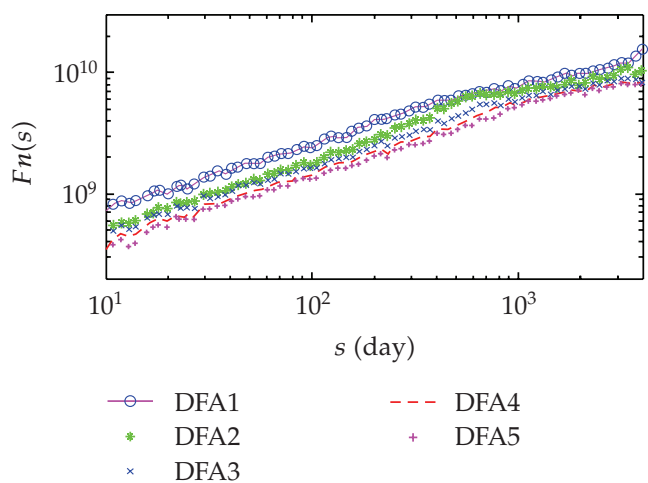

(f)

Figure 4: DFA of the series with correlated trend $A[X(i)]^{p}$, that is, $Y(i)=X(i)+A[X(i)]^{p}$, where $A=1$, (a) $p=1.8$. (b) $p=1$.9. (c) $p=2.8$. (d) $p=2.9$. (e) $p=3.8$. (f) $p=3$.9. (a) $\rightarrow$ (b) is the process that crossover $s_{1 x}^{(1)}$ changes from $84(<100)$ to $106(>100)$. (c) $\rightarrow$ (d) is the "vanishing" process of $s_{1 x}^{(1)}$ and $s_{1 x}^{(2)}$ while $(\mathrm{e}) \rightarrow(\mathrm{f})$ is the "vanishing" process of $s_{1 x}^{(n)}, n=3,4,5$. Some typical crossovers are tagged by arrows.

get the final stable property around $p=n-0.1$, close to $n$. It may be related to the fact that an $(n+1)$ th DFA can eliminate an $n$th order polynomial trend due to the integration in DFA algorithm.

(3) $p<1$

As we can see from Figure 3(a), when $p=1$, that the extra trend $A[X(i)]^{p}$ does not have any influence on the correlation properties of the series. And as most data in our original 


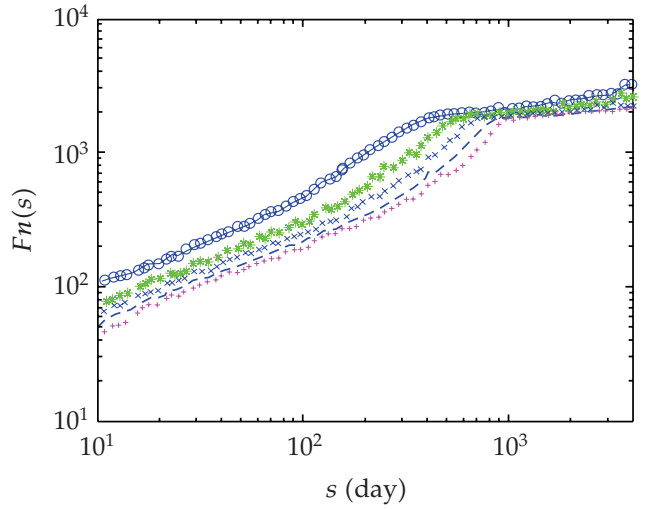

(a)

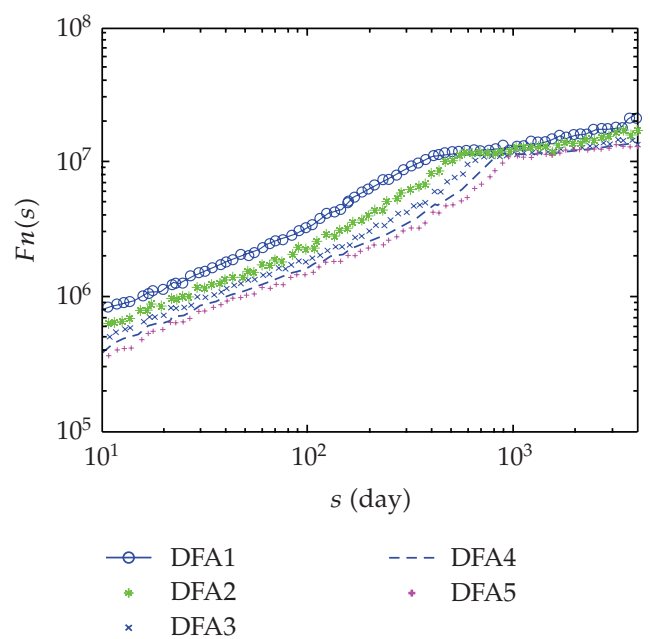

(c)

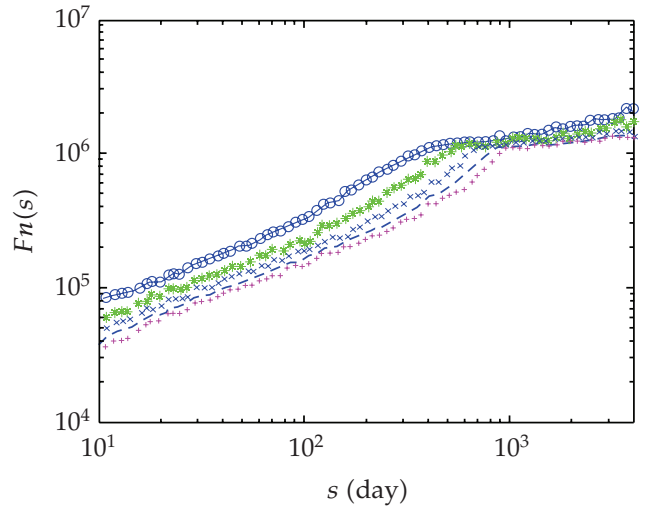

(b)

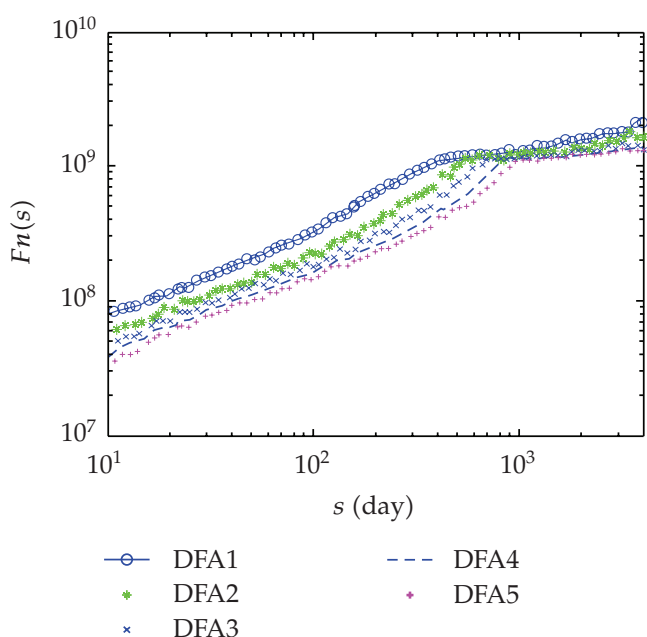

(d)

Figure 5: DFA of the series with correlated trend $A[X(i)]^{p}$, that is, $Y(i)=X(i)+A[X(i)]^{p}$ where $p=2$, (a) $A=0.01$, (b) $A=10$, (c) $A=100$, (d) $A=10000$.

precipitation, series are larger than $1.0(0.1 \mathrm{~mm})$. We can guess that when $p<1$, that is, the adding correlated trend is weaker than that of one above, the correlated trend does not affect the correlation properties either and we prove it in Table 4.

We find from Table 4 that the crossovers $s_{x}^{(n)}$ of $p<1$ are just the same as the original data. They are rather close to the original data as well by observing their scaling exponents. The trends are so weak that there is little influence of $A[X(i)]^{p}$ on the correlation properties of $X(i)$.

\subsubsection{Effect of coefficient $A$ on DFA of $Y(i)$}

In Section 4.2.1, we have studied the correlation properties of the series with correlated trend $A[X(i)]^{p}$, where $A$ is a constant 1 . And we find that the crossovers $s_{2 x}^{(n)}$ are independent of power $p$ of trend $A[X(i)]^{p}$. So how about coefficient $A$ ? 


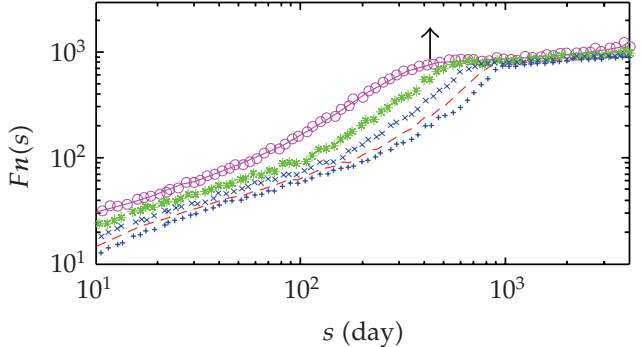

(a)

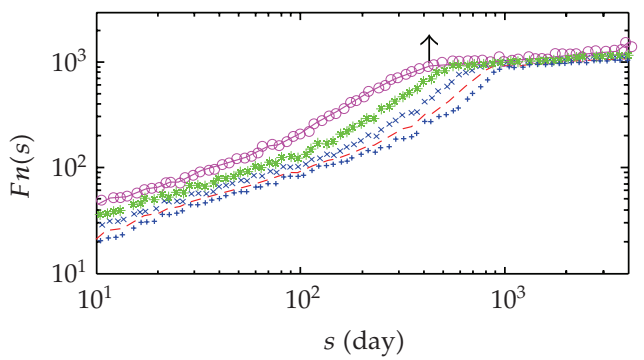

(c)

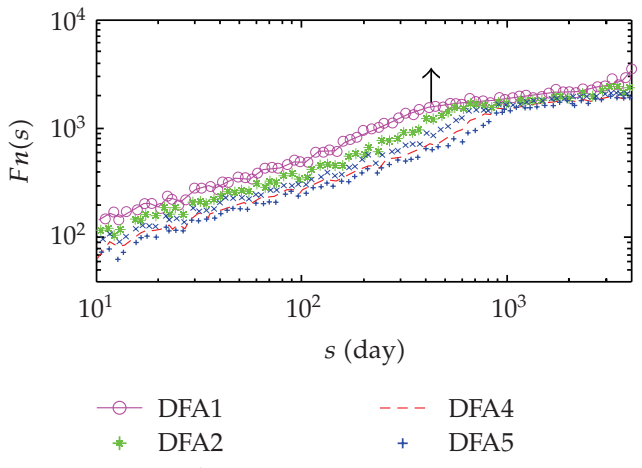

(e)

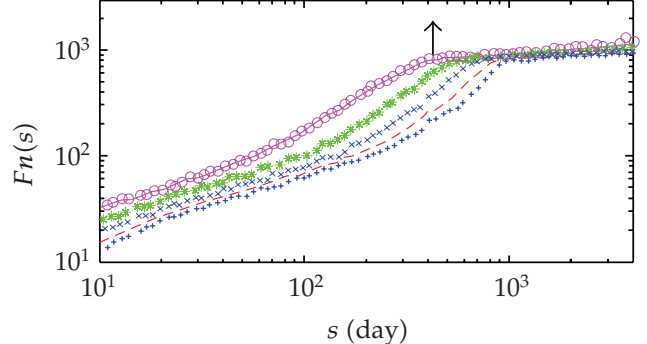

(b)

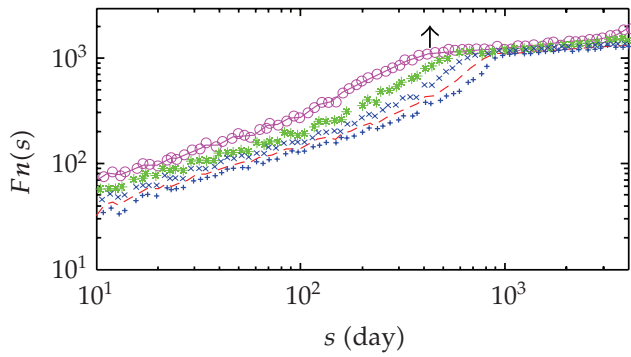

(d)

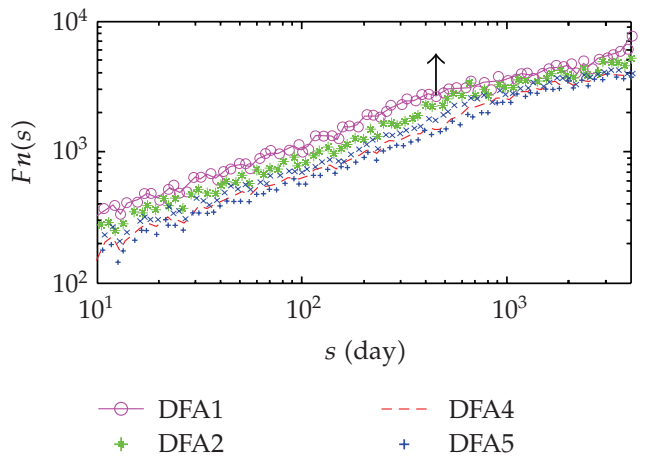

(f)

Figure 6: DFA of the series with correlated trend $A e^{p X(i) / \bar{x}}$, where $A=10$, (a) $p=2$, (b) $p=3$, (c) $p=5$, (d) $p=6$, (e) $p=7$, (f) $p=8$.

In this section we vary $A$ to find the relation between $A$ and the correlation properties of $Y(i)$. As the correlation properties for DFA of $Y(i)$ in Section 4.2.1 is the most complicated when $p=2$, here we just test coefficient $A$ with $p=2$.

There is a clear vision that the crossover times of (a), (b), (c), and (d) are just the same as Figure 3(b). And the scaling exponents $\alpha^{(n)}$ seem to be also the same that we exhibit them in Table 5 to make it evident. The scaling exponents $\alpha^{(n)}$ of Figures 5(a), 5(b), 5(c), and $5(\mathrm{~d})$ are not exactly the same by values but they hold the same scaling behavior with Figure 3(b).When $A$ is large, the scaling exponents $\alpha^{(n)}$ are rather close to the ones of $A=1$ in Figure 3(b). The crossovers in DFA result from the competition between the scaling of original series and the scaling of the trend. The case of $A<0$ is similar to that of $A>0$ which can be inferred by the following analysis. If the original series dominates the scaling behavior, apparently the symbol of $A$ can be negligible. When the added trends prevail, most 
Table 4: DFA of the series with correlated trend $A[X(i)]^{p}$, that is, $Y(i)=X(i)+A[X(i)]^{p} p<1$. We pick four values of $p$ as below.

\begin{tabular}{|c|c|c|c|c|c|c|}
\hline & & DFA1 & DFA2 & DFA3 & DFA4 & DFA5 \\
\hline \multirow{5}{*}{$p=-3$} & $\alpha_{0}^{(n)}$ & 0.6713 & 0.6261 & 0.6121 & 0.6317 & 0.6414 \\
\hline & $s_{1 x}^{(n)}$ & 84 & 98 & 146 & 234 & 274 \\
\hline & $\alpha_{1}^{(n)}$ & 1.1376 & 1.2730 & 1.3603 & 1.4800 & 1.4869 \\
\hline & $s_{2 x}^{(n)}$ & 406 & 556 & 704 & 892 & 965 \\
\hline & $\alpha_{2}^{(n)}$ & 0.1524 & 0.1429 & 0.1242 & 0.1267 & 0.1299 \\
\hline \multirow{5}{*}{$P=-1$} & $\alpha_{0}^{(n)}$ & 0.6710 & 0.6260 & 0.6120 & 0.6315 & 0.6412 \\
\hline & $s_{1 x}^{(n)}$ & 84 & 98 & 146 & 234 & 274 \\
\hline & $\alpha_{1}^{(n)}$ & 1.1364 & 1.2713 & 1.3584 & 1.4778 & 1.4847 \\
\hline & $s_{2 x}^{(n)}$ & 406 & 556 & 704 & 892 & 965 \\
\hline & $\alpha_{2}^{(n)}$ & 0.1530 & 0.1434 & 0.1245 & 0.1270 & 0.1301 \\
\hline \multirow{5}{*}{$P=-0.5$} & $\alpha_{0}^{(n)}$ & 0.6711 & 0.6260 & 0.6120 & 0.6315 & 0.6412 \\
\hline & $s_{1 x}^{(n)}$ & 84 & 98 & 146 & 234 & 274 \\
\hline & $\alpha_{1}^{(n)}$ & 1.1371 & 1.2722 & 1.3594 & 1.4791 & 1.4860 \\
\hline & $s_{2 x}^{(n)}$ & 406 & 556 & 704 & 892 & 965 \\
\hline & $\alpha_{2}^{(n)}$ & 0.1527 & 0.1432 & 0.1244 & 0.1269 & 0.1300 \\
\hline \multirow{5}{*}{$p=0.5$} & $\alpha_{0}^{(n)}$ & 0.6746 & 0.6276 & 0.6131 & 0.6330 & 0.6429 \\
\hline & $s_{1 x}^{(n)}$ & 84 & 98 & 146 & 234 & 274 \\
\hline & $\alpha_{1}^{(n)}$ & 1.1522 & 1.2937 & 1.3835 & 1.5068 & 1.5143 \\
\hline & $s_{2 x}^{(n)}$ & 406 & 556 & 704 & 892 & 965 \\
\hline & $\alpha_{2}^{(n)}$ & 0.1460 & 0.1379 & 0.1210 & 0.1244 & 0.1284 \\
\hline
\end{tabular}

Table 5: Scaling exponents of Figures 5(a), 5(b), 5(c), and 5(d).

\begin{tabular}{lllllll}
\hline$P=2$ & & DFA1 & DFA2 & DFA3 & DFA4 & DFA5 \\
\hline \multirow{4}{*}{$A=0.01$} & $\alpha_{0}^{(n)}$ & 0.6425 & 0.6045 & 0.5947 & 0.6081 & 0.6183 \\
& $\alpha_{1}^{(n)}$ & 0.9691 & 1.0743 & 1.1243 & 1.2200 & 1.2169 \\
& $\alpha_{2}^{(n)}$ & 0.2182 & 0.1884 & 0.1457 & 0.1404 & 0.1388 \\
\hline \multirow{3}{*}{$A=10$} & $\alpha_{0}^{(n)}$ & 0.6150 & 0.5926 & 0.5855 & 0.5965 & 0.6072 \\
& $\alpha_{1}^{(n)}$ & 0.8718 & 0.9434 & 0.9871 & 1.0621 & 1.0571 \\
& $\alpha_{2}^{(n)}$ & 0.2687 & 0.2262 & 0.1691 & 0.1586 & 0.1547 \\
\hline \multirow{3}{*}{$A=100$} & $\alpha_{0}^{(n)}$ & 0.6150 & 0.5925 & 0.5855 & 0.5964 & 0.6071 \\
& $\alpha_{1}^{(n)}$ & 0.8717 & 0.9432 & 0.9869 & 1.0619 & 1.0569 \\
& $\alpha_{2}^{(n)}$ & 0.2688 & 0.2263 & 0.1692 & 0.1586 & 0.1547 \\
\hline \multirow{4}{*}{$A=10000$} & $\alpha_{0}^{(n)}$ & 0.6150 & 0.5925 & 0.5855 & 0.5964 & 0.6071 \\
& $\alpha_{1}^{(n)}$ & 0.8717 & 0.9432 & 0.9869 & 1.0619 & 1.0568 \\
& $\alpha_{2}^{(n)}$ & 0.2688 & 0.2263 & 0.1692 & 0.1586 & 0.1547 \\
\hline
\end{tabular}




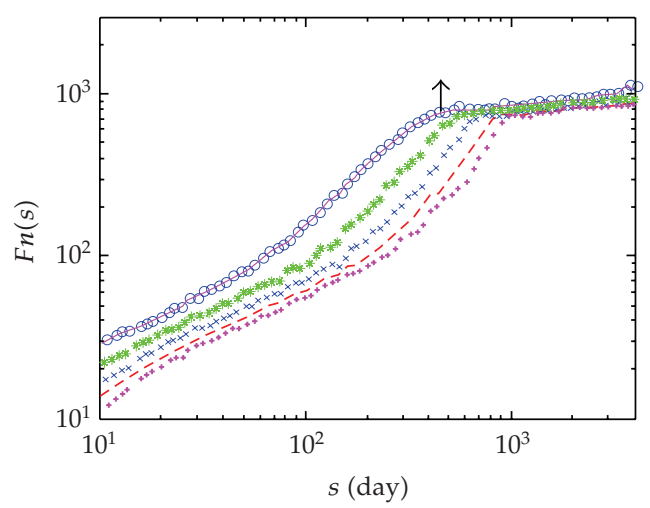

(a)

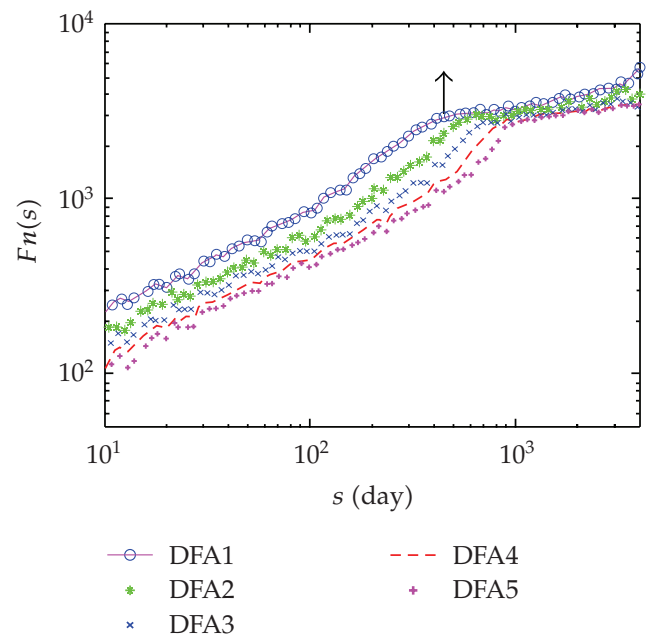

(c)

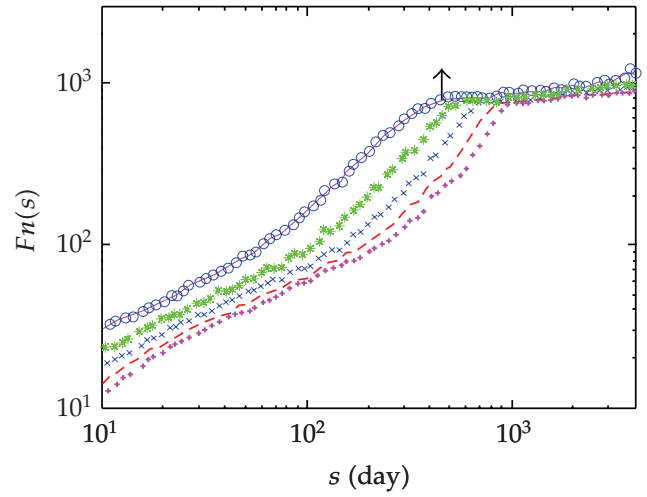

(b)

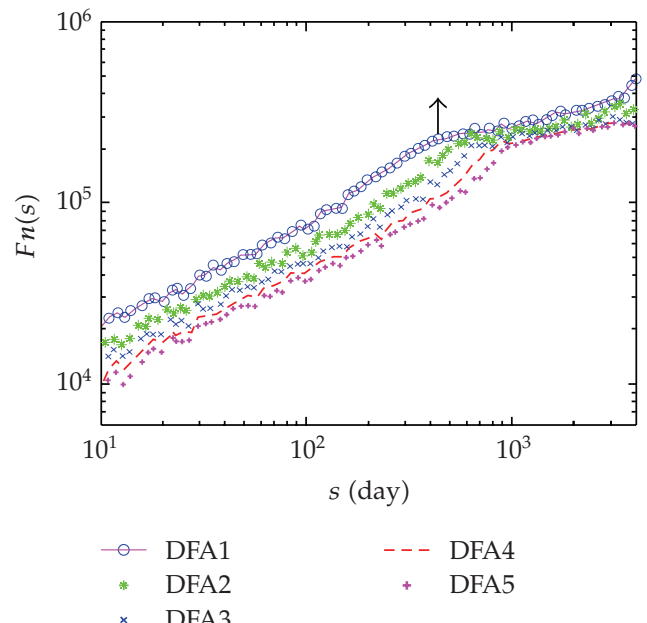

(d)

Figure 7: DFA of the series with correlated trend $A e^{p X(i) / \bar{x}}$, where $p=5$, (a) $A=0.01$, (b) $A=1$, (c) $A=100$, (d) $A=10000$.

values may change the signs, but the magnitude of fluctuation and the period of trend remain unchangeable. Of course, $A=-1$ and $p=1$ should be excluded. So the correlation properties with correlated trend $A[X(i)]^{p}$ are independent of the coefficient $A$.

\subsection{DFA of the Series with Other Correlated Trends}

Discussion of Section 2 has told that crossovers $s_{2 x}^{(n)}$ of the original data are independent of the trend $A[X(i)]^{p}$ which presents another question which is are they still independent of other correlated trends? In this section we test another common trend $A e^{p X(i) / \bar{x}}(\bar{x}$ is the max of $X(i)$ ) which is stronger than $A[X(i)]^{p}$ with the same method applied in Section 2. We test the effects of $A$ and $p$ on the correlation properties of $Z(i)=X(i)+A e^{p X(i) / \bar{x}}$, respectively, in Figures 6 and 7.

Although Figure 7 indicates that the correlation properties of series with correlated trend $A e^{p X(i) / \bar{x}}$ are dependent of $A$ which are not the same as they are for trend $A[X(i)]^{p}$, 
we can see form Figures 6 and 7 that the crossover times $s_{2 x}^{(n)}$ are just identical to the ones of original series for each figure. So crossovers $s_{2 x}^{(n)}$ of the original data are independent of the correlated trend $A e^{p X(i) / \bar{x}}$. It is an obvious conclusion that crossovers $s_{2 x}^{(n)}$ are independent of trend $A \log (p X(i))$ as well, because it is weaker than $A[X(i)]^{p}$, not to mention $A e^{p X(i) / \bar{x}}$. Thus we can see that crossovers $s_{2 x}^{(n)}$ of the original data are rather significant and they are so stable that it is necessary to take further investigations to gain insight into them.

\section{Conclusion}

In summary, DFA of the original series indicates the complex correlation properties by two obvious crossovers and three different scaling segments. The second crossover $s_{2 x}^{(n)}$ is proved to be independent of the adding correlated types of trend: $A \log (p X(i)), A[X(i)]^{p}$, and $A e^{p X(i) / \bar{x}}$, while the first crossover $s_{1 x}^{(n)}$ disappears with added trend. They are induced by different reasons while behave similarly if we just analyze original precipitation series. The paper also provides a method to distinguish the multifractal and trend effects on the scaling behavior. With the development of study on correlation, scaling exponents and crossovers will take more significant roles in providing foundation theories for precipitation series predictions based on correlations theories.

\section{Acknowledgments}

The financial supports from the funds of The National High Technology Research Development Program of China (863 Program) (2007AA11Z212), the China National Science (60772036) and MEDF (20070004002) are gratefully acknowledged.

\section{References}

[1] C.-K. Peng, S. V. Buldyrev, S. Havlin, M. Simons, H. E. Stanley, and A. L. Goldberger, "Mosaic organization of DNA nucleotides," Physical Review E, vol. 49, no. 2, pp. 1685-1689, 1994.

[2] C.-K. Peng, J. E. Mietus, Y. Liu et al., "Quantifying fractal dynamics of human respiration: age and gender effects," Annals of Biomedical Engineering, vol. 30, no. 5, pp. 683-692, 2002.

[3] J. W. Kantelhardt, S. A. Zschiegner, E. Koscielny-Bunde, S. Havlin, A. Bunde, and H. E. Stanley, "Multifractal detrended fluctuation analysis of nonstationary time series," Physica A, vol. 316, no. 1-4, pp. 87-114, 2002.

[4] K. Hu, P. C. Ivanov, Z. Chen, P. Carpena, and H. E. Stanley, "Effect of trends on detrended fluctuation analysis," Physical Review E, vol. 64, no. 1, Article ID 011114, 19 pages, 2001.

[5] Z. Chen, P. C. Ivanov, K. Hu, and H. E. Stanley, "Effect of nonstationarities on detrended fluctuation analysis," Physical Review E, vol. 65, no. 4, Article ID 041107, 15 pages, 2002.

[6] Z. Chen, K. Hu, P. Carpena, P. Bernaola-Galvan, H. E. Stanley, and P. C. Ivanov, “Effect of nonlinear filters on detrended fluctuation analysis," Physical Review E, vol. 71, no. 1, Article ID 011104, 11 pages, 2005.

[7] R. Nagarajan and R. G. Kavasseri, "Minimizing the effect of periodic and quasi-periodic trends in detrended fluctuation analysis," Chaos, Solitons and Fractals, vol. 26, no. 3, pp. 777-784, 2005.

[8] P. Shang, Y. Lu, and S. Kamae, "Detecting long-range correlations of traffic time series with multifractal detrended fluctuation analysis," Chaos, Solitons and Fractals, vol. 36, no. 1, pp. 82-90, 2008.

[9] P. Shang, A. Lin, and L. Liu, "Chaotic SVD method for minimizing the effect of exponential trends in detrended fluctuation analysis," Physica A, vol. 388, no. 5, pp. 720-726, 2009.

[10] Ming Li and Wei Zhao, "Representation of a stochastic traffic bound," IEEE Transactions on Parallel and Distributed Systems. In press. 
[11] M. Li and S. C. Lim, "Modeling network traffic using generalized Cauchy process," Physica A, vol. 387, no. 11, pp. 2584-2594, 2008.

[12] M. Li, "Generation of teletraffic of generalized Cauchy type," Physica Scripta, vol. 81, no. 2, Article ID 025007, 2010.

[13] M. Li and J.-Y. Li, "On the predictability of long-range dependent series," Mathematical Problems in Engineering, vol. 2010, Article ID 397454, 9 pages, 2010.

[14] G. Mattioli, M. Scalia, and C. Cattani, "Analysis of large amplitude pulses in short time intervals: application to neuron interactions," accepted to Mathematical Problems in Engineering.

[15] C.-Y. Chu and K. S. Chen, "Effects of rain fading on the efficiency of the Ka-band LMDS system in the Taiwan area," IEEE Transactions on Vehicular Technology, vol. 54, no. 1, pp. 9-19, 2005.

[16] R. K. Crane, "Prediction of attenuation by rain," IEEE Transactions on Communications Systems, vol. 28, no. 9, pp. 1717-1733, 1980.

[17] R. K. Crane, "A local model for the prediction of rain-rate statistics for rain-attenuation models," IEEE Transactions on Antennas and Propagation, vol. 51, no. 9, pp. 2260-2273, 2003.

[18] K. Fraedrich and C. Larnder, "Scaling regimes of composite rainfall time series," Tellus, Series A, vol. 45, no. 4, pp. 289-298, 1993.

[19] D. Harris, M. Menabde, A. Seed, and G. Austin, "Multifractal characterization of rain fields with a strong orographic influence," Journal of Geophysical Research D, vol. 101, no. 21, pp. 26405-26414, 1996.

[20] S. Lovejoy and D. Schertzer, "Multifractals and rain," in New Uncertainty Concepts in Hydrology and Water Resources, pp. 61-103, Cambridge University Press, Cambridge, UK, 1995.

[21] C. Matsoukas, S. Islam, and I. Rodriguez-Iturbe, "Detrended fluctuation analysis of rainfall and streamflow time series," Journal of Geophysical Research, vol. 105, pp. 29165-29172, 2000.

[22] M. Li, "Fractal time series-a tutorial review," Mathematical Problems in Engineering, vol. 2010, Article ID 157264, 26 pages, 2010.

[23] J. W. Kantelhardt, E. Koscielny-Bunde, H. H. A. Rego, S. Havlin, and A. Bunde, “Detecting long-range correlations with detrended fluctuation analysis," Physica A, vol. 295, no. 3-4, pp. 441-454, 2001.

[24] S. Kimiagar, M. S. Movahed, S. Khorram, S. Sobhanian, and M. R. R. Tabar, "Fractal analysis of discharge current fluctuations," Journal of Statistical Mechanics: Theory and Experiment, vol. 2009, no. 3, Article ID P03020, 2009.

[25] G. Toma, "Specific differential equations for generating pulse sequences," Mathematical Problems in Engineering, vol. 2010, Article ID 324818, 11 pages, 2010.

[26] E. G. Bakhoum and C. Toma, "Dynamical aspects of macroscopic and quantum transitions due to coherence function and time series events," Mathematical Problems in Engineering, vol. 2010, Article ID 428903, 2010.

[27] H. A. Makse, S. Havlin, M. Schwartz, and H. E. Stanley, "Method for generating long-range correlations for large systems," Physical Review E, vol. 53, no. 5, pp. 5445-5449, 1996.

[28] B. Podobnik, P. C. Ivanov, K. Biljakovic, D. Horvatic, H. E. Stanley, and I. Grosse, "Fractionally integrated process with power-law correlations in variables and magnitudes," Physical Review E, vol. 72, no. 2, Article ID 026121, 7 pages, 2005.

[29] I. M. Jánosi and R. Müller, "Empirical mode decomposition and correlation properties of long daily ozone records," Physical Review E, vol. 71, no. 5, Article ID 056126, 5 pages, 2005.

[30] X.-Y. Qian, W.-X. Zhou, and G.-F. Gu, "Modified detrended fluctuation analysis based on empirical mode decomposition," http://arxiv.org/abs/0907.3284.

[31] S. Y. Chen, Y. F. Li, and J. Zhang, "Vision processing for realtime 3-D data acquisition based on coded structured light," IEEE Transactions on Image Processing, vol. 17, no. 2, pp. 167-176, 2008.

[32] S. Y. Chen, Y. F. Li, Q. Guan, and G. Xiao, "Real-time three-dimensional surface measurement by color encoded light projection," Applied Physics Letters, vol. 89, no. 11, Article ID 111108, 2006.

[33] J.-R. Yeh, S.-Z. Fan, and J.-S. Shieh, "Human heart beat analysis using a modified algorithm of detrended fluctuation analysis based on empirical mode decomposition," Medical Engineering and Physics, vol. 31, no. 1, pp. 92-100, 2009.

[34] C. V. Chianca, A. Ticona, and T. J. P. Penna, "Fourier-detrended fluctuation analysis," Physica A, vol. 357, no. 3-4, pp. 447-454, 2005.

[35] C. Cattani and A. Kudreyko, "Application of periodized harmonic wavelets towards solution of eigenvalue problems for integral equations," Mathematical Problems in Engineering, vol. 2010, Article ID 570136, 8 pages, 2010. 


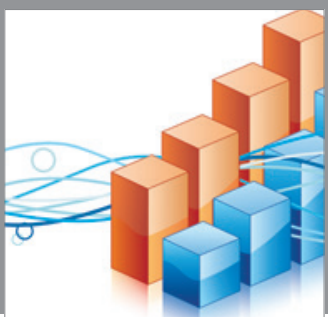

Advances in

Operations Research

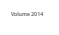

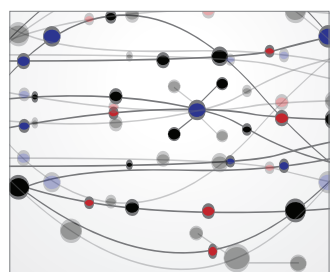

\section{The Scientific} World Journal
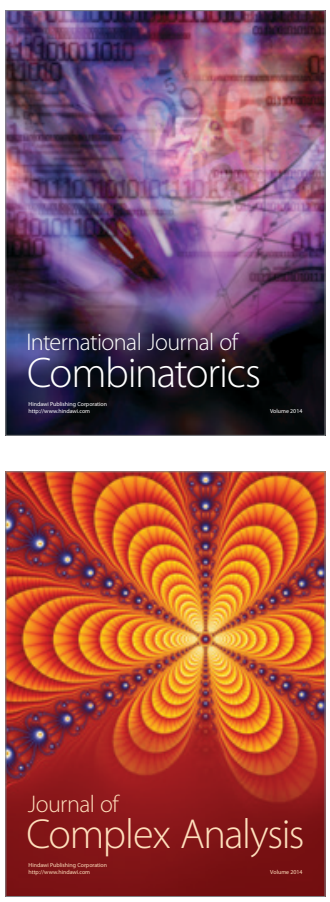

International Journal of

Mathematics and

Mathematical

Sciences
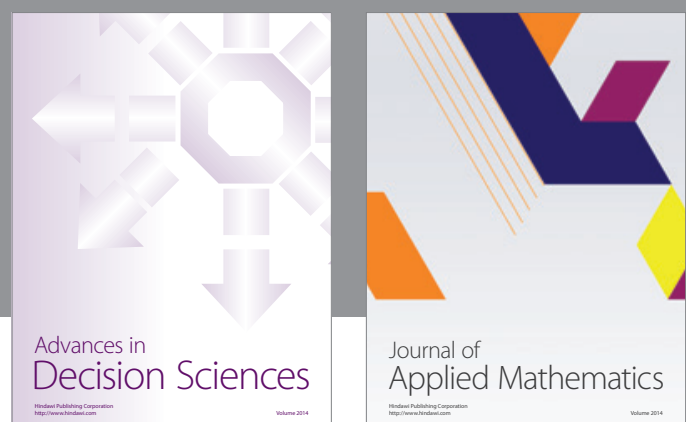

Journal of

Applied Mathematics
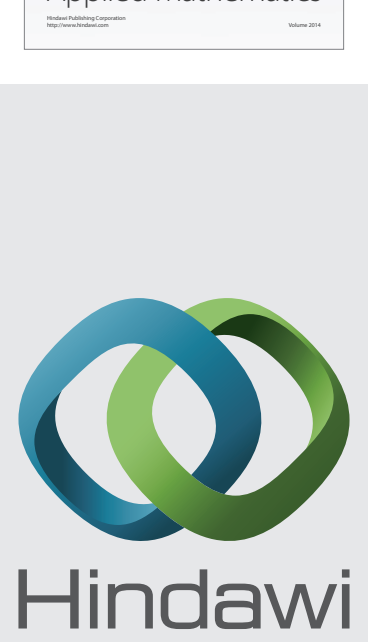

Submit your manuscripts at http://www.hindawi.com
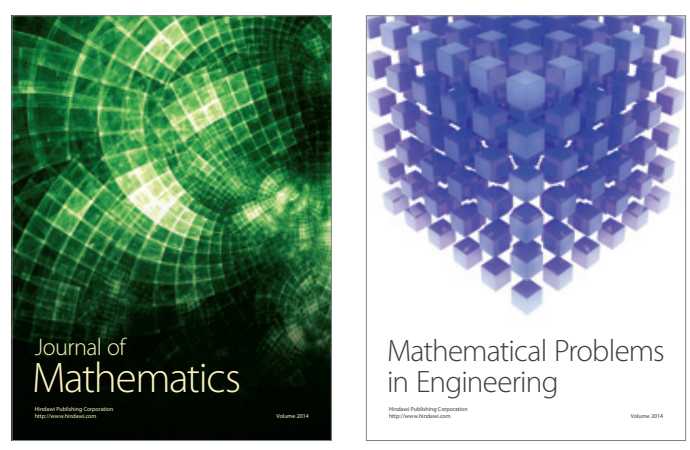

Mathematical Problems in Engineering
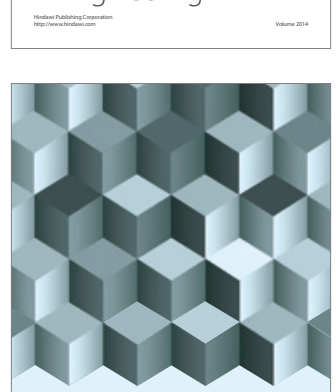

Journal of

Function Spaces
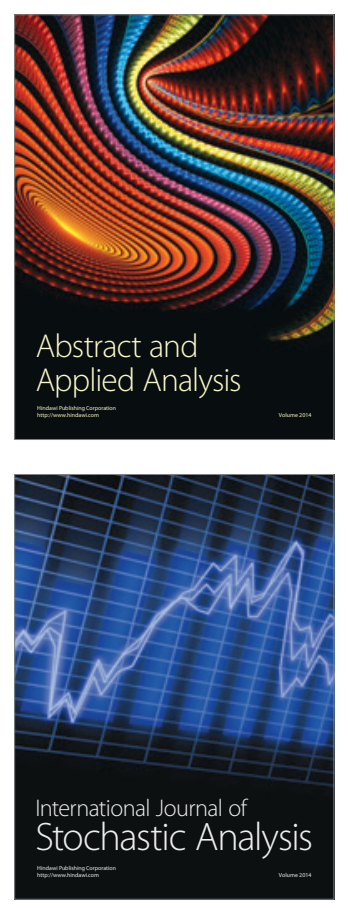

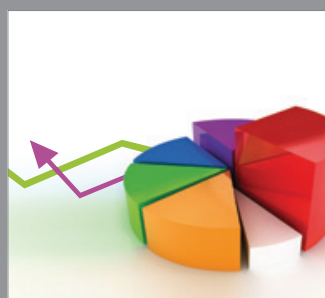

ournal of

Probability and Statistics

Promensencen
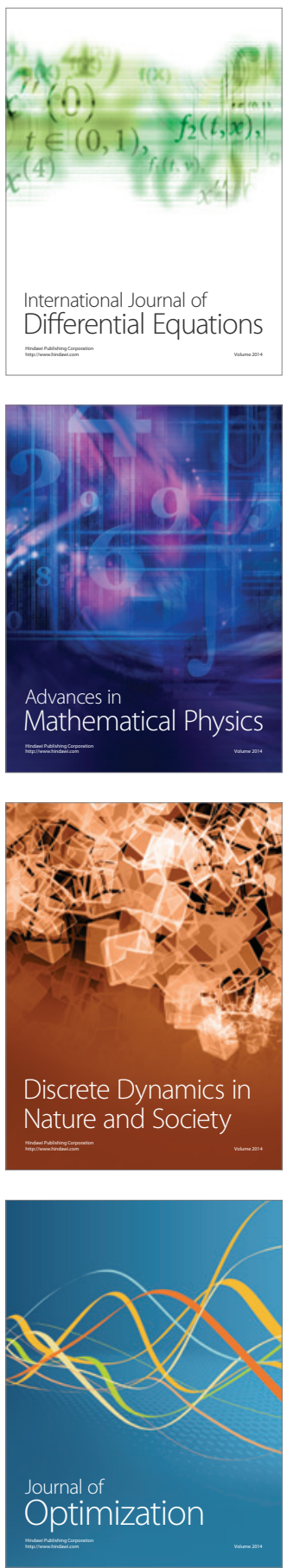